\title{
Double Ionization of Unsaturated Hydrocarbons Interacting with High-Power Femtosecond Laser Pulses
}

\author{
S. M. Sharifi, ${ }^{1}$ A. Talebpour, ${ }^{1,2}$ and S. L. Chin ${ }^{1}$ \\ ${ }^{1}$ Département de Physique, de Génie Physique et d'Optique, Centre d'Optique, Photonique et Laser (COPL), \\ Université Laval, QC, Canada G1K 7P4 \\ ${ }^{2}$ Novx Systems Inc., 185 Renfrew Dr., Markham, ON, Canada L3R 6 G3
}

Correspondence should be addressed to S. M. Sharifi, s-mehdi.sharifi.1@ulaval.ca

Received 28 September 2009; Accepted 16 December 2009

Recommended by Dong-Sheng Guo

The nonsequential double ionization of four unsaturated organic molecules, $\mathrm{C}_{5} \mathrm{NH}_{5}, \mathrm{C}_{3} \mathrm{H}_{6}, \mathrm{C}_{2} \mathrm{H}_{4}$, and $\mathrm{C}_{2} \mathrm{H}_{2}$ interacting with strong Ti: Sapphire laser pulses are reported. Despite differences in the molecular geometry, the degree of ionization suppression and the fraction of molecules that undergo non-sequential double ionization were similar for all of these molecules.

Copyright ( 2009 S. M. Sharifi et al. This is an open access article distributed under the Creative Commons Attribution License, which permits unrestricted use, distribution, and reproduction in any medium, provided the original work is properly cited.

\section{Introduction}

In recent years, some studies on the occurrence of nonsequential double ionization in hydrocarbons have been reported. Kosmidis et al. [1] attributed the observation of doubly charged ions in the case of aromatic molecules like Pyridine to the sequential channel. Obviously, their ions versus peak laser intensity plots have not been prepared over a sufficiently large dynamic range to concretely rule out the possibility of nonsequential double ionization. Cornaggia and Hering [2] have studied the laser induced ionization of four molecules $\mathrm{N}_{2}, \mathrm{CO}_{2}, \mathrm{C}_{2} \mathrm{H}_{2}$, and $\mathrm{C}_{3} \mathrm{H}_{4}$ in great detail, and presented unambiguous evidences of the occurrence of nonsequential double ionization. For example, they observed that the ratio $\left[\mathrm{C}_{2} \mathrm{H}_{2}^{2+}\right] /\left[\mathrm{C}_{2} \mathrm{H}_{2}^{+}\right]$, at laser intensities below the saturation intensity of singly charged ion, is a slowly increasing function of intensity when linearly polarized pulses are employed, while the same ratio steeply drops by lowering intensity for circularly polarized pulses. This implies a huge enhancement in the rate of doubly charged ion production at lower intensities. According to the arguments put forward by Talebpour et al. in connection with the multiple ionization of $\mathrm{Kr}$ and $\mathrm{Xe}$ [3], this enhancement can be taken as an indication of the dominance of nonsequential double ionization. More recently, $\mathrm{Ma}$ et al. [4] studied the double ionization of $\mathrm{C}_{2} \mathrm{H}_{4}$ and $\mathrm{C}_{2} \mathrm{H}_{6}$ and concluded that these molecules undergo nonsequential double ionization even in the case of circularly polarized light. Unfortunately, the details of experimental setup and, more importantly, the method of calibrating the laser intensity were not provided. Therefore, their conclusions appear doubtful in contrast to the results of Cornaggia and Hering [2]. As it can be judged from the small number of publications on a subject dealing with a large set of molecules with varying structures, the studies have not been sufficient to conclude a generalized and quantitative trend in the dependency of the nonsequential ionization of hydrocarbons on the laser intensity. Interestingly, the corresponding formulation is already in place for rare gas atoms, which was established through experimental [5] and theoretical [6] studies. According to these results, the observed doubly charged ion signal of an atom $A$ at the peak laser intensity $I, S_{\text {obs }}\left(A^{2+}, I\right)$, can be calculated from the following relation:

$$
S_{\text {obs }}\left(A^{2+}, I\right)=S_{\mathrm{PPT}}\left(A^{2+}, I\right)+\alpha_{12} S_{\mathrm{ADK}}\left(A^{+}, I\right),
$$

where $S_{\mathrm{PPT}}\left(A^{2+}, I\right)$ is the sequential ion yield predicted by the PPT [7] or, equivalently, the Krainov models $[8,9]$ and the laser wavelength-dependent quantity $\alpha_{12}$ is the ratio of neutral atoms that ionize through the direct nonsequential channel $A \stackrel{\text { non-sequential }}{\longrightarrow} A^{++}$to the atoms that ionize via quasistatic tunneling, $A \stackrel{\text { QS-tunnelling }}{\longrightarrow} A^{+}$. It is well known that the rate of the quasistatic tunneling process is satisfactorily given by the ADK formula [10]; thus we 
approximated $S_{\mathrm{QS} \text {-tunnelling }}\left(A^{+}, I\right)$ with $S_{\mathrm{ADk}}\left(A^{+}, I\right)$. It should be noted that (1) does not specify the mechanism responsible for the nonsequential ionization. About ten years ago, after successfully fitting the experimental results on the nonsequential ionization of atoms by a rescattering based formalism in reference [6], the rescattering mechanism appeared to be the mechanism responsible for the nonsequential ionization. In the case of nonsequential double ionization of molecules, the question of mechanism has not been settled. However, the shake-up excitation during optical ionization has recently been shown to account for the nonsequential ionization of $\mathrm{D}_{2}$ [11]. More recently, $\mathrm{Wu}$ et al. [12] have attributed the double ionization of $\mathrm{CH}_{4}$ to the electron rescattering.

It is plausible that a trend similar to the one predicted by relation (1) be applicable to the case of unsaturated organic molecules. This was explicitly demonstrated in our recent publication [13] in the case of benzene. We found that the double ionization of benzene interacting with linearly polarized Ti: Sapphire laser pulses occurs mainly through nonsequential processes. Benzene exhibits unusually large relative probability for nonsequential double ionization which was tentatively attributed to the suppression of multiphoton/tunnel ionization to singly charged ions. This is interesting when it is noted that a hydrocarbon molecule suffers a noticeable suppression of over one order of magnitude in ionization to singly charged ion [14]. Thus, a thorough examination of double ionization phenomenon in unsaturated organic molecules can be important for clarifying if there is interplay between the ionization suppression and the nonsequential ionization. We undertake such a study in this article by considering the laser-induced ionization of four molecules; $\mathrm{C}_{2} \mathrm{H}_{2}, \mathrm{C}_{2} \mathrm{H}_{4}, \mathrm{C}_{3} \mathrm{H}_{6}$, and $\mathrm{C}_{5} \mathrm{NH}_{5}$. Our selection has been based on the following reasons. All these molecules undergo suppressed ionization to the singly charged states. Furthermore, $\mathrm{C}_{2} \mathrm{H}_{4}$ has been observed to undergo ionization suppression similar to $\mathrm{C}_{5} \mathrm{NH}_{5}$ [15] noting that their ionization to singly charged ion can be described by employing the same value of the quantity $Z_{\text {eff }}$ [16]. This parameter is an empirically determined quantity that approximates the ionization suppression by assuming the tunneling of electron from a $Z_{\text {eff }} / r$ barrier instead of purely Coulombic barrier of $1 / r$ used in the case of rare gas atoms. Despite the foretold similarity the geometry of the two molecules is vastly different to the level that remarkably different cross sections for the ionization by the rescattered electrons [17] are expected to influence the probability of double ionization. Indeed, any observed difference can be discussed by comparing with the case of $\mathrm{C}_{3} \mathrm{H}_{6}$, an asymmetric molecule, to verify if the structural symmetries influence the probability of double ionization or suppression. Finally, the case of $\mathrm{C}_{2} \mathrm{H}_{2}$ with very strong $\mathrm{C} \equiv \mathrm{C}$ bond will address the effects of bond strength.

\section{Experimental Setup}

The experimental setup has already been described in reference [5]. Briefly, a Ti: Sapphire laser system provides a train of 800-nm transform limited pulses having a fullwidth-at-half-maximum time duration of $45 \mathrm{fs}$. The linearly polarized pulses were focused using a lens with a focal length of $100 \mathrm{~cm}$ into a high vacuum chamber having a background pressure of $5 \times 10^{-9}$ Torr. Ion species were separated with a time-of-flight mass spectrometer and ion curves were produced by combining a series of intensity scans, each having a different fill pressure in the interaction chamber ranging from $10^{-9}$ to $10^{-5}$ Torr. In order to estimate the absolute peak intensity at the focal region we followed the technique employed in [13]: near the saturation region, the experimental result and ADK theory for $\mathrm{He}^{+}$signal in the Ti: sapphire laser field has an excellent overlap. We calibrated the absolute intensity based on a comparison with the saturation intensity predicted by the ADK formula using Helium in the vacuum chamber.

\section{Experimental Results and Discussion}

In the following sub-sections, information on the ionization potential of the studied neutral and singly charged ions will be needed for quantitative discussion of the results. Therefore, we summarize this information in Table 1. We use these ionization potential data for theoretical calculation using the PPT model and the ADK model for all molecules. All this data are from electron impact ionization for the first ionization energy $\mathrm{Ip}^{(+)}$and the second ionization energy $\mathrm{Ip}^{(++)}$.

3.1. Pyridine $\left(\mathrm{C}_{5} \mathrm{H}_{5} \mathrm{~N}\right)$. The ion yield versus peak intensity plots of $\mathrm{C}_{5} \mathrm{H}_{5} \mathrm{~N}^{+}$and $\mathrm{C}_{5} \mathrm{H}_{5} \mathrm{~N}^{++}$along with four theoretically calculated curves are presented in Figure 1. The data are more scattered than the corresponding data in the case of other species, which will be presented in the following subsections. This scattering is due to the fact that Pyridine is liquid and it is difficult to maintain a constant gas pressure in the interaction chamber during intensity scan.

Before discussing the details of these curves, we should clarify an important issue. The curve denoted by $S_{\text {PPT }}\left(\mathrm{C}_{5} \mathrm{H}_{5} \mathrm{~N}^{+}\right)$in Figure 1 has been calculated by assuming a multiphoton/tunneling rate given by the PPT model with the effective charge acting on ionizing electron, $Z_{\text {eff }}$, as a free parameter. This technique, evidently, is semiempirical in nature for the quantity $Z_{\text {eff }}$ includes the effects of parameters like the orbital structure of the molecule, orientation of the molecule with respect to laser polarization as well as charge distribution on atomic centers. Interestingly, this single quantity is sufficient to fit the experimentally observed ion yield with predictions of a theory intended for simple $1 / r$ Columbic potential. This is the basis of approximating a complex polyatomic molecule with an atom like species. Here, we do not go in the details of the subject as there have been some justifications for the validity of this approximation in a paper by our group [18]. Recently, Uiterwaal et al. [19], based on a new approach to intense-field ionization of atoms and molecules, haven shown that the apparent ionization suppression of some molecules are due to their lower single-photon absorption 
TABLE 1: First and second ionization potential (Ip) of the studied molecules.

\begin{tabular}{lcccc}
\hline & $\mathrm{C}_{2} \mathrm{H}_{2}$ & $\mathrm{C}_{2} \mathrm{H}_{4}$ & $\mathrm{C}_{3} \mathrm{H}_{6}$ & $\mathrm{C}_{5} \mathrm{H}_{5} \mathrm{~N}$ \\
\hline $\mathrm{Ip}^{(+)}(\mathrm{ev})$ & 11.4 & 10.5 & 9.75 & 9.3 \\
$\mathrm{Ip}^{(++)}(\mathrm{ev})$ & $32.7[20]$ & $31.2[21]$ & $28.3[21]$ & $27.1[22]$ \\
\hline
\end{tabular}

cross-section compared to the case of atoms. Another interesting observation is that we found the same value of 0.78 for $Z_{\text {eff }}$ as reference [18] in the case of Benzene even though the pulse duration in our case is nearly 5 times shorter for Pyridine. The quantity $S_{\mathrm{PPT}}\left(\mathrm{C}_{5} \mathrm{H}_{5} \mathrm{~N}^{+}\right)$presents the total rate of the laser induced ionization disregarding the fact that this ionization occurs via one of the two possible channels: ionization by absorbing multiple photons or tunneling through a quasistatic barrier (solid line fitted to singly charge ion). The rate of tunneling process is given by $S_{\mathrm{ADK}}\left(\mathrm{C}_{5} \mathrm{H}_{5} \mathrm{~N}^{+}\right)$and $Z_{\text {eff }}=0.78$. Multiplying $S_{\mathrm{ADK}}\left(\mathrm{C}_{5} \mathrm{H}_{5} \mathrm{~N}^{+}\right)$ by a factor $\alpha_{12}=0.02$ a curve is obtained (dot line) that satisfactorily fits the observed signal of the doubly charged ions at intensities lower than the saturation intensity of the singly charged ions $\left(1 \times 10^{14} \mathrm{~W} / \mathrm{cm}^{2}\right)$. This is equivalent to saying that the ionization process leading to doubly charged ion at lower intensities is nonsequential in nature and follows relation 1. It should be mentioned that $S_{\mathrm{ADK}}\left(\mathrm{C}_{5} \mathrm{H}_{5} \mathrm{~N}^{+}\right)$was calculated by setting $Z_{\text {eff }}=0.78$. This value of $Z_{\text {eff }}$ was chosen since the saturation of nonsequential doubly charged signal (represented by $\alpha_{12} S_{\mathrm{ADK}}\left(\mathrm{C}_{5} \mathrm{H}_{5} \mathrm{~N}^{+}\right)$) and the singly charged ion occurred at the same laser intensity.

At higher intensities the sequential double ionization, predicted by $S_{\mathrm{PPT}}\left(\mathrm{C}_{5} \mathrm{H}_{5} \mathrm{~N}^{++}\right)$(dash line) cannot find any experimental data to fit. In fact, only nonsequential ionization curve is almost sufficient to fit the data. At the high intensity end, the experimental signal is slightly higher than predicted by the nonsequential curve. This means that there is a little sequential ionization going on at higher intensity, but cannot be fitted by the full curve $S_{\text {obs }}$ (dashdot line), which is calculated according to relation 1 . The latter overestimates the measured ion signal in the sequential part. The conclusion is that, similar to the case of rare gas atoms, relation 1 is able to predict the double ionization of Pyridine. The only difference is that an empirically determined $Z_{\text {eff }}$ must be used when calculating the ionization rates in PPT and ADK model. In addition, at higher intensity fragmentation dominates the interaction channel and most of the doubly charged ions do not survive. Therefore, the contribution of the sequential double ionization, predicted by the PPT model, is barely noticeable on the experimentally observed doubly charged ion.

3.2. Propene $\left(\mathrm{C}_{3} \mathrm{H}_{6}\right)$. The ion yield versus peak intensity plots of $\mathrm{C}_{3} \mathrm{H}_{6}^{+}$and $\mathrm{C}_{3} \mathrm{H}_{6}^{++}$along with four theoretically calculated curves are presented in Figure 2. The curve denoted by $S_{\mathrm{PPT}}\left(\mathrm{C}_{3} \mathrm{H}_{6}^{+}\right)$in the Figure 2 has been calculated by setting the value of the effective charge, $Z_{\text {eff, }}$ at 0.78 . Interestingly, this value is the same as in the case of Pyridine, indicating the details of molecular geometry do not affect the ionization probability to a measureable amount (solid line fitted to

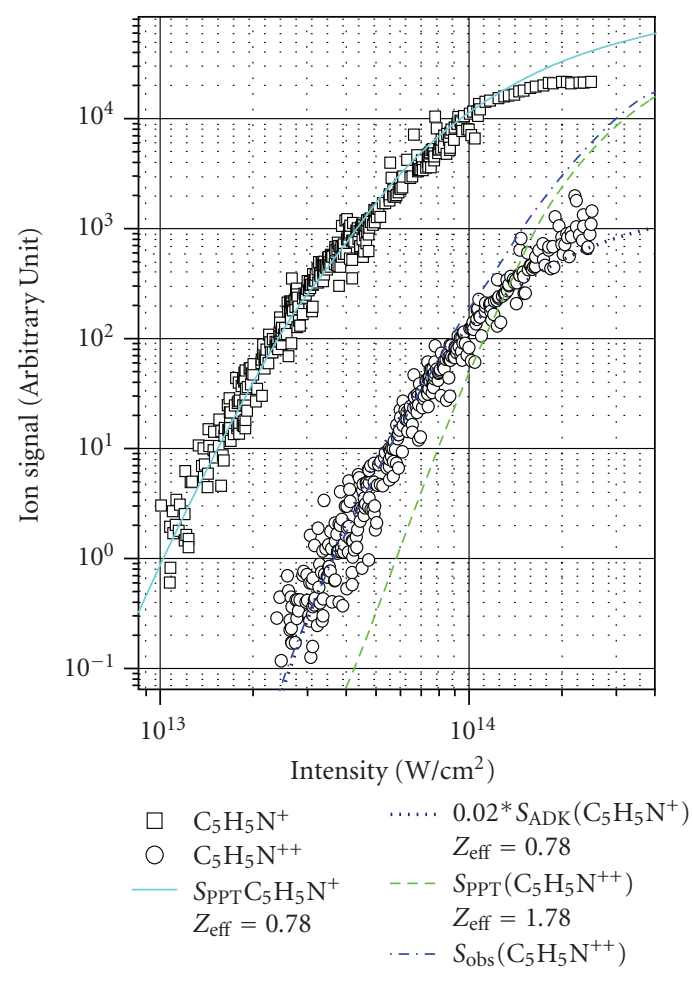

FIGURE 1: The ion yield versus peak intensity plots of $\left(\mathrm{C}_{5} \mathrm{NH}_{5}{ }^{+}\right)$ and $\left(\mathrm{C}_{5} \mathrm{NH}_{5}{ }^{++}\right)$along with four theoretically calculated curves are presented. The $S_{\text {obs }}$ is obtained according to relation (1).

singly charge ion). Multiplying $S_{\mathrm{ADK}}\left(\mathrm{C}_{3} \mathrm{H}_{6}^{+}\right)$by a factor $\alpha_{12}=$ 0.02 a curve is obtained that satisfactorily fits (dot line) the observed signal of the doubly charged ions at intensities lower than the saturation intensity of the singly charged ions $\left(1.5 \times 10^{14} \mathrm{~W} / \mathrm{cm}^{2}\right)$. This is very similar to the nonsequential ionization of Pyridine where the same $\alpha_{12}=0.02$ was found; that is, their probabilities of nonsequential ionization are similar even though their shapes and symmetries are very different. At higher intensities, sequential double ionization, predicted by $S_{\mathrm{PPT}}\left(\mathrm{C}_{3} \mathrm{H}_{6}^{++}\right)$, should dominate (dash line). As it can be seen, the experimental data indicates a continued increase of the doubly charged ions but fall short of following the $S_{\mathrm{PPT}}\left(\mathrm{C}_{3} \mathrm{H}_{6}^{++}\right)$. This would mean that, unlike benzene and Pyridine, some sequential double ionization occurs before fragmentation. This is equivalent to saying that the ionization process leading to doubly charged ion at lower intensities is nonsequential in nature and in the higher intensity we have some sequential double ionization. The reason for the observation of this sequential ionization could be because it is more difficult to break (fragment) the molecule as compared to Pyridine and benzene in the same intensity range. However, this sequential ionization signal is lower than the predicted total signal shown by the curve $S_{\text {obs }}$ (dash-dot line) in Figure 2, which is calculated according to relation (1). In conclusion, even though the shape and symmetries of Propene and Pyridine are very different, the probability of nonsequential double ionization is found to be similar. 


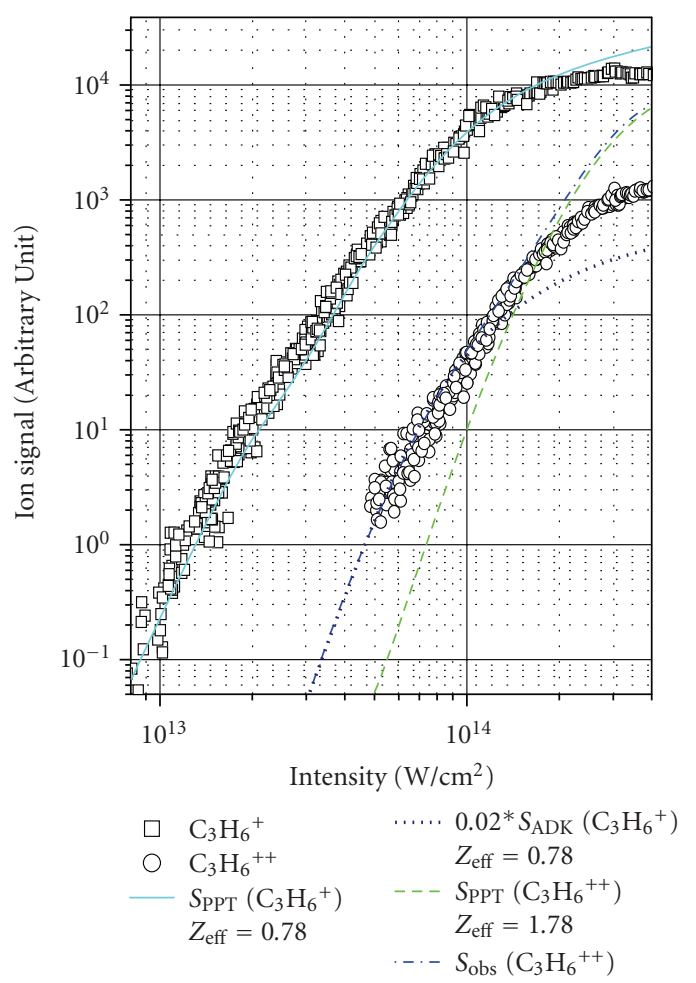

FIgURE 2: The ion yield versus peak intensity plots of $\mathrm{C}_{3} \mathrm{H}_{6}^{+}$and $\mathrm{C}_{3} \mathrm{H}_{6}^{++}$along with four theoretically calculated curves are presented.

3.3. Ethylene $\left(\mathrm{C}_{2} \mathrm{H}_{4}\right)$. The ion yield versus peak intensity plots of $\mathrm{C}_{2} \mathrm{H}_{4}^{+}$and $\mathrm{C}_{2} \mathrm{H}_{4}^{++}$along with four theoretically calculated curves are presented in Figure 3. The explanations of all theoretically calculated curves are the same as Pyridine and Propene. Since $\mathrm{C}_{2} \mathrm{H}_{4}^{++}$and $\mathrm{CH}_{2}^{+}$ions have identical mass to charge ratio, the observed ion signal at $m / q=14$ is a combination of the two ion signals and one may argue that the ion yield plot of Figure 3 over estimates the amount of doubly charged ions. However, in what follows, by applying a fragmentation mechanism proposed by our group $[15,18]$, it will show that the contribution of $\mathrm{CH}_{2}^{+}$coming from the dissociation of the singly charged ion cannot exceed $10 \%$.

According to the foretold fragmentation model, unsaturated hydrocarbons interacting with strong laser pulses fragment via a three step process. (a) An electron from an inner orbital of the molecule is ejected and results in a molecular ion in ro-vibrational levels of an excited electronic state; (b) the exited ion makes a rapid radiationless transition to the high-lying ro-vibrational levels of a lower electronic state; (c) the ion dissociates to different fragments through various fragmentation channels. Therefore, the abundance of a particular ionic fragment is determined by the ionization probability of the inner valance electrons. The appearance potential of $\mathrm{CH}_{2}^{+}$ion, that is, the minimum energy needed to form the fragment from a neutral molecule in its ground state, has been measured to be $18.4 \mathrm{eV}$ [23]. In the presence of laser field, this will be reduced by $\Delta E \approx 0.5 F R$, where $R$ is the size of ion and $F$ is the strength of the field [24]. For example at an intensity of $6 \times 10^{13} \mathrm{~W} / \mathrm{cm}^{2}$ and assuming

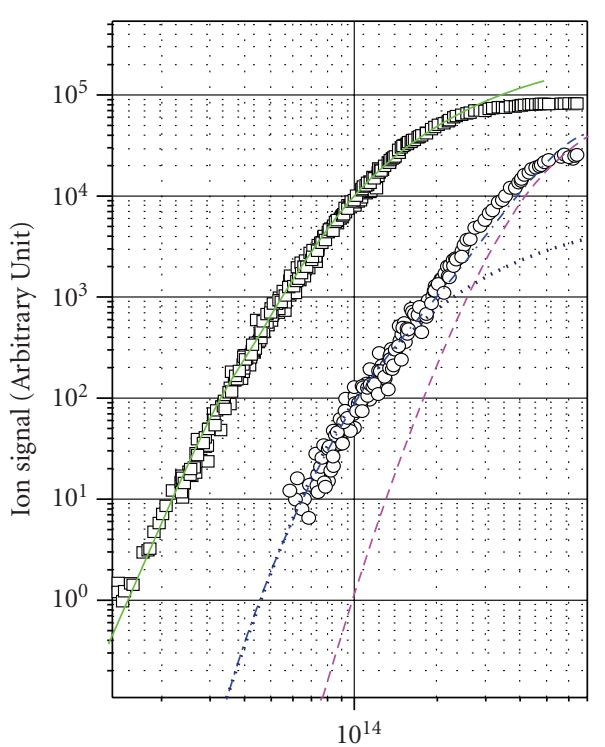

Intensity $\left(\mathrm{W} / \mathrm{cm}^{2}\right)$

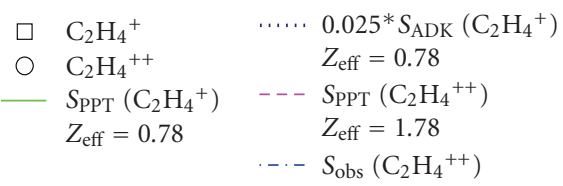

Figure 3: The ion yield versus peak intensity plots of $\mathrm{C}_{2} \mathrm{H}_{4}^{+}$and $\mathrm{C}_{2} \mathrm{H}_{4}^{++}$along with four theoretically calculated curves are presented.

the size of $R \sim 2 \mathrm{au}, \Delta E$ is around $1.1 \mathrm{eV}$. The value of $\Delta E$ at $6 \times 10^{14} \mathrm{~W} / \mathrm{cm}^{2}$, the highest intensity at which we have performed the present experiment, amounts to $3.48 \mathrm{eV}$. Therefore the appearance potential in the intensity range of our experiments varies from 17.3 to $14.92 \mathrm{eV}$. By calculating the probability of inner-valence ionization and assuming that all ionized ions undergo dissociation process, we found that the theoretically estimated $\mathrm{CH}_{2}^{+}$ion signal is at least one order of magnitude lower than the observed signal at $m / q=14$. Therefore, we will equate this peak with doubly charged ion in lower intensity and nonsequential part. Of course, this statement is not true at higher intensities $(I>$ $2 \times 10^{14} \mathrm{~W} / \mathrm{cm}^{2}$ ) where the fragmentation of doubly charged ions dominate the dissociation process. In this region if the second electron emerging from the molecular ion comes from breaking the $\left(\pi_{\mathrm{C}=\mathrm{C}}\right)$ bond [25], $\mathrm{C}_{2} \mathrm{H}_{4}^{++}$could be broken through the channel: $\mathrm{C}_{2} \mathrm{H}_{4}^{++} \rightarrow \mathrm{CH}_{2}^{+}+\mathrm{CH}_{2}^{+}$and this process could increase the ion signal at $m / q=14$. This is manifested in the following. The curve denoted by $S_{\mathrm{PPT}}\left(\mathrm{C}_{2} \mathrm{H}_{4}^{+}\right)$in Figure 3 has been calculated by setting $Z_{\text {eff }}=0.78$. This value is similar to those used in Pyridine and Propene meaning that the degree of suppression is the same. In order to fit the nonsequential part of $\mathrm{C}_{2} \mathrm{H}_{4}^{++}$, we have multiplied $S_{\mathrm{ADK}}\left(\mathrm{C}_{2} \mathrm{H}_{4}^{+}\right)$by a factor $\alpha_{12}=0.025$. This value is similar to the value in the case of Pyridine and Porpene. However, the calculated sequential ionization $S_{\mathrm{PPT}}\left(\mathrm{C}_{2} \mathrm{H}_{4}^{++}\right)$and hence the calculated total ionization $S_{\text {obs }}$ is lower than the experimental data in the sequential part of doubly charged ion signal indicating some extra contribution from the dissociation of 


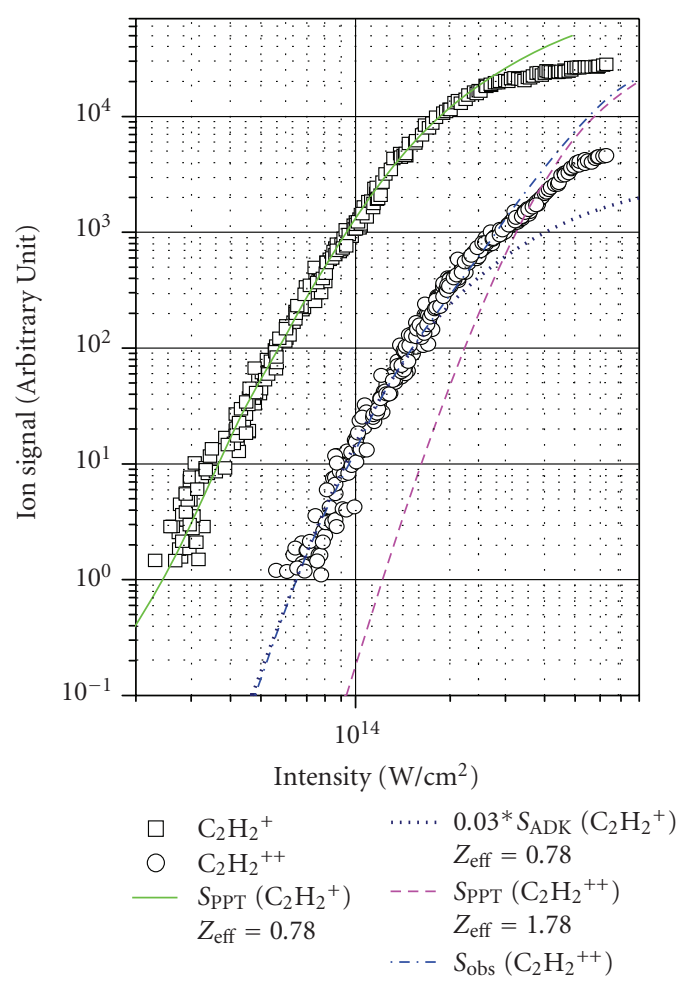

FIgURE 4: The ion yield versus peak intensity plots of $\mathrm{C}_{2} \mathrm{H}_{2}^{+}$and $\mathrm{C}_{2} \mathrm{H}_{2}^{++}$along with four theoretically calculated curves are presented.

$\mathrm{C}_{2} \mathrm{H}_{4}^{++}$into $\mathrm{C}_{2} \mathrm{H}_{2}^{+}$as discussed above. Thus, the probability of nonsequential double ionization of Ethylene is found to be similar to the two foretold molecules. But in the sequential part (high intensity region) there is some contribution of the fragmentation of molecular doubly charged ion in the sequential ion signal.

3.4. Acetylene $\left(\mathrm{C}_{2} \mathrm{H}_{2}\right)$. The ion yield versus peak intensity plots of $\mathrm{C}_{2} \mathrm{H}_{2}^{+}$and $\mathrm{C}_{2} \mathrm{H}_{2}^{++}$along with three theoretically calculated curves are presented in Figure 4. The explanation of all theoretically calculated curves is the same as Pyridine, Propene and ethylene. In this case of Acetylene, we have the same issue as Ethylene. Since $\mathrm{C}_{2} \mathrm{H}_{2}^{++}$and $\mathrm{CH}^{+}$ions have identical mass to charge ratio, the observed ion signal at $m / q=13$ is a combination of the two ion signals and one may argue that the ion yield plot of Figure 4 over estimates the amount of doubly charged ions. However, an argument similar to the case of Ethylene will show that the contribution of $\mathrm{CH}^{+}$on the observed ion peak in the nonsequential part cannot exceed $10 \%$. The appearance potential of $\mathrm{CH}^{+}$ion from neutral $\mathrm{C}_{2} \mathrm{H}_{2}$ has been measured to be $20.8 \mathrm{eV}$ by electron impact [26]; this should be compared to that of Ethylene which is $18.4 \mathrm{eV}$ [19]. Indeed, similar to Cornaggia et al. [27] only two molecular ion components $\mathrm{C}_{2} \mathrm{H}_{2}^{+}$and $\mathrm{C}_{2} \mathrm{H}_{2}^{++}$could be seen in the mass spectrum. According to Cornaggia et al. [27], the absence, or very weak contribution to the total ion signal, of molecular ion species such as $\mathrm{C}_{2} \mathrm{H}^{+}$ and $\mathrm{CH}_{2}^{+}$means that the fragmentation channels involving these ions are not produced during the laser interaction.
This in turn would mean that the probability of dissociation into $\mathrm{CH}^{+}$ion is low. This is understandable noting that two carbon atoms in $\mathrm{C}_{2} \mathrm{H}_{2}$ are bound by a triple bound in contrast to double bound in $\mathrm{C}_{2} \mathrm{H}_{4}$. In this case Acetylene we found the $\alpha_{12}=0.02$. We thus conclude that the probability of nonsequential double ionization of acetylene is found to be similar to the three foretold molecules. But in the sequential part, some sequential ionization occurs before fragmentation. The degree of sequential ionization seems to vary from molecule to molecule.

\section{Conclusion}

The ionization of four unsaturated organic molecules, $\mathrm{C}_{5} \mathrm{NH}_{5}, \mathrm{C}_{3} \mathrm{H}_{6}, \mathrm{C}_{2} \mathrm{H}_{4}$, and $\mathrm{C}_{2} \mathrm{H}_{2}$, interacting with strong linearly polarized $\mathrm{Ti}$, Sapphire laser pulses, was studied. Two phenomena were observed. The first is that the probability of ionization to the singly charged ion is suppressed compare to fictitious atoms with the same ionization potentials. The degrees of suppression represented by the $Z_{\text {eff }}$ values are similar for all of them despite the differences in the molecular geometry. The second is that, similar to benzene, double ionization of these molecules occurs mainly through the nonsequential process. These molecules exhibit unusually large relative probability for nonsequential ionization which was attributed to the suppression of multiphoton/tunnel ionization to singly charged ions. In addition, it was observed that the probability of nonsequential double ionization is similar in these molecules as reflected by the similarity of the value $\alpha_{12}$ in spite of the difference in molecular geometry.

\section{References}

[1] C. Kosmidis, P. Tzallas, K. W. D. Ledingham, et al., "Multiply charged intact Ions of polyatomic cyclic molecules generated by a strong laser field," Journal of Physical Chemistry A, vol. 103, no. 35, pp. 6950-6955, 1999.

[2] C. Cornaggia and P. Hering, "Nonsequential double ionization of small molecules induced by a femtosecond laser field," Physical Review A, vol. 62, no. 2, Article ID 023403, 13 pages, 2000.

[3] A. Talebpour, C. Y. Chien, Y. Liang, and S. L. Chin, "Nonsequential ionization of $\mathrm{Xe}$ and $\mathrm{Kr}$ in an intense femtosecond Ti:sapphire laser pulse," Journal of Physics B, vol. 30, no. 7, pp. 1721-1730, 1997.

[4] R. Ma, C. Wu, J. Huang, et al., "Double ionization of $\mathrm{C}_{2} \mathrm{H}_{4}$ and $\mathrm{C}_{2} \mathrm{H}_{6}$ molecules irradiated by an intense femtosecond laser field," Chemical Physics Letters, vol. 404, no. 4-6, pp. 370-373, 2005.

[5] S. Larochelle, A. Talebpour, and S. L. Chin, "Non-sequential multiple ionization of rare gas atoms in a Ti:sapphire laser field," Journal of Physics B, vol. 31, no. 6, pp. 1201-1214, 1998.

[6] A. Becker and F. H. M. Faisal, "Production of high-charge states of Xe in a femtosecond laser pulse," Physical Review A, vol. 59, no. 5, pp. R3182-R3185, 1999.

[7] A. M. Perelemov and V. S. Popov, "Ionization of atoms in alternating electric field. III," Soviet Physics. JETP, vol. 25, pp. 336-343, 1967.

[8] V. P. Krainov, "Ionization rates and energy and angular distributions at the barrier-suppression ionization of complex 
atoms and atomic ions," JOSA B, vol. 14, no. 2, pp. 425-431, 1997.

[9] S. Larochelle, A. Talebpour, and S. L. Chin, "Coulomb effect in multiphoton ionization of rare-gas atoms," Journal of Physics B, vol. 31, no. 6, pp. 1215-1224, 1998.

[10] M. V. Ammosov, N. B. Delone, and V. P. Krainov, “Tunnel ionization of complex atoms and atomic ions in an alternating electromagnetic field," Soviet Physics, vol. 64, pp. 1191-1194, 1986.

[11] I. V. Litvinyuk, F. Légaré, P. W. Dooley, D. M. Villeneuve, and P. B. Corkum, "Shakeup excitation during optical tunnel ionization,” Physical Review Letter, vol. 94, no. 3, Article ID 033003, 2005.

[12] Z. Wu, C. Wu, Q. Liang, et al., "Fragmentation dynamics of methane by few-cycle femtosecond laser pulses," Journal of Chemical Physics, vol. 126, no. 7, Article ID 074311, 2007.

[13] S. M. Sharifi, A. Talebpour, and S. L. Chin, "Double ionization of benzene interacting with strong Ti:sapphire laser pulses," Journal of Physics B, vol. 102, p. 259, 2007.

[14] A. Talebpour, S. Larochelle, and S. L. Chin, "Multiphoton ionization of unsaturated hydrocarbons," Journal of Physics B, vol. 31, no. 12, pp. 2769-2776, 1998.

[15] A. Talebpour, A. D. Bandrauk, J. Yang, and S. L. Chin, "Multiphoton ionization of inner-valence electrons and fragmentation of ethylene in an intense Ti:sapphire laser pulse," Chemical Physics Letters, vol. 313, no. 5-6, pp. 789-794, 1999.

[16] A. Talebpour, S. Larochelle, and S. L. Chin, "Suppressed tunnelling ionization of the D-2 molecule in an intense Ti : sapphire laser pulse," Journal of Physics B, vol. 31, no. 2, pp. L49-L58, 1998.

[17] P. B. Corkum, "Plasma perspective on strong field multiphoton ionization," Physical Review Letters, vol. 71, no. 13, pp. 1994-1997, 1993.

[18] A. Talebpour, A. D. Bandrauk, and S. L. Chin, "Fragmentation of benzene in an intense $\mathrm{Ti}$ : sapphire laser pulse," Laser Physics, vol. 10, no. 1, pp. 210-215, 2000.

[19] C. J. G. J. Uiterwaal, C. R. Gebhardt, H. Schroder, and K. L. Kompa, "Predicting intense-field photoionization of atoms and molecules from their linear photoabsorption spectra in the ionization continuum," European Physical Journal D, vol. 30, no. 3, pp. 379-392, 2004.

[20] N. Jeffreys, S. R. Andrews, D. E. Parry, and F. M. Harris, "Double ionization of the ethyne molecule," Rapid Communications in Mass Spectrometry, vol. 10, no. 13, pp. 1693-1697, 1996.

[21] B. Brehm, U. Frobe, and H. P. Neitzke, "Doubly charged ions. Mass spectra and appearance energies for small hydrocarbons," The International Journal of Mass Spectrometry, vol. 57, no. 1, pp. 91-102, 1984.

[22] K. Vekey and G. Brenton, "Electron capture-induced decomposition of the pyridine $\left|; \mathrm{C}_{5} \mathrm{H}_{5} \mathrm{~N}\right| ;^{2+}$ ion," The International Journal of Mass Spectrometry, vol. 87, no. 1, pp. 121-133, 1989.

[23] I. H. Suzuki and K. Maeda, "Ionization efficiency curves and fragmentations of deuterated ethylenes by electron impact," International Journal of Mass Spectrometry and Ion Physics, vol. 15, no. 3, pp. 281-290, 1974.

[24] A. D. Bandrauk, Molecules in Laser Field, chapter 3, Marcel Dekker, Inc., New York, NY, USA, 1994.

[25] T. Ibuki, T. Koyano, T. Masuoka, and C. E. Brion, "Dissociation of doubly charged $\mathrm{CH}_{2}=\mathrm{CD}_{2}$ and $\mathrm{CH}_{2}=\mathrm{CF}_{2}$ in the region of valence shell photoexcitation," The Journal of Chemical Physics, vol. 98, no. 4, pp. 2908-2915, 1993.
[26] S. J. King and S. D. Price, "Electron ionization of acetylene," Journal of Chemical Physics, vol. 127, no. 17, Article ID 174307, 16 pages, 2007.

[27] C. Cornaggia, M. Schmidt, and D. Normand, "Laser-induced nuclear motions in the Coulomb explosion of $\mathrm{C}_{2} \mathrm{H}_{2}^{+}$ions," Physical Review A, vol. 51, no. 2, pp. 1431-1437, 1995. 

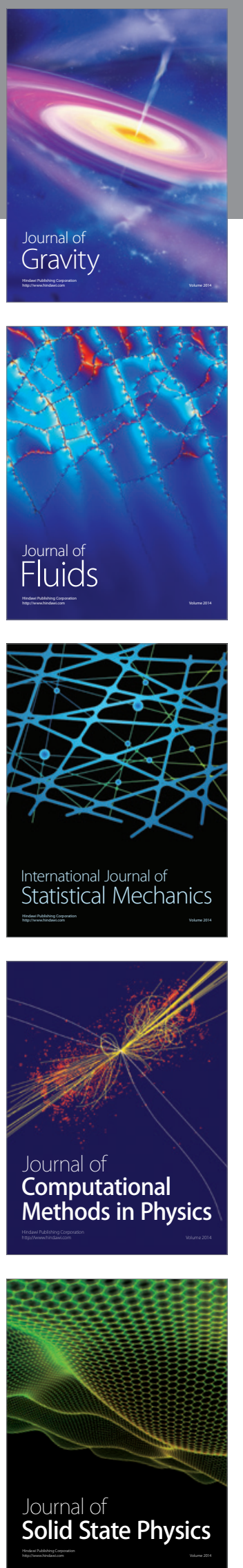

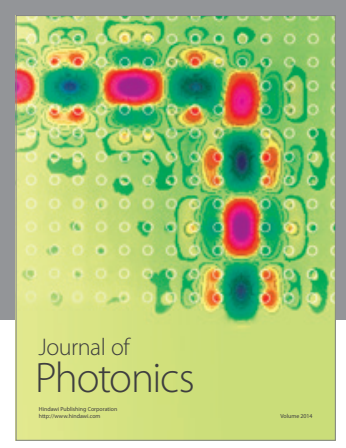

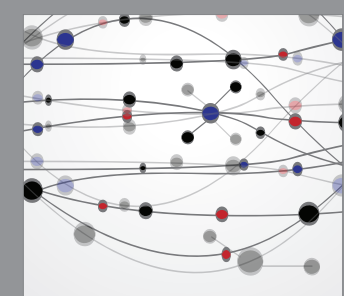

The Scientific World Journal
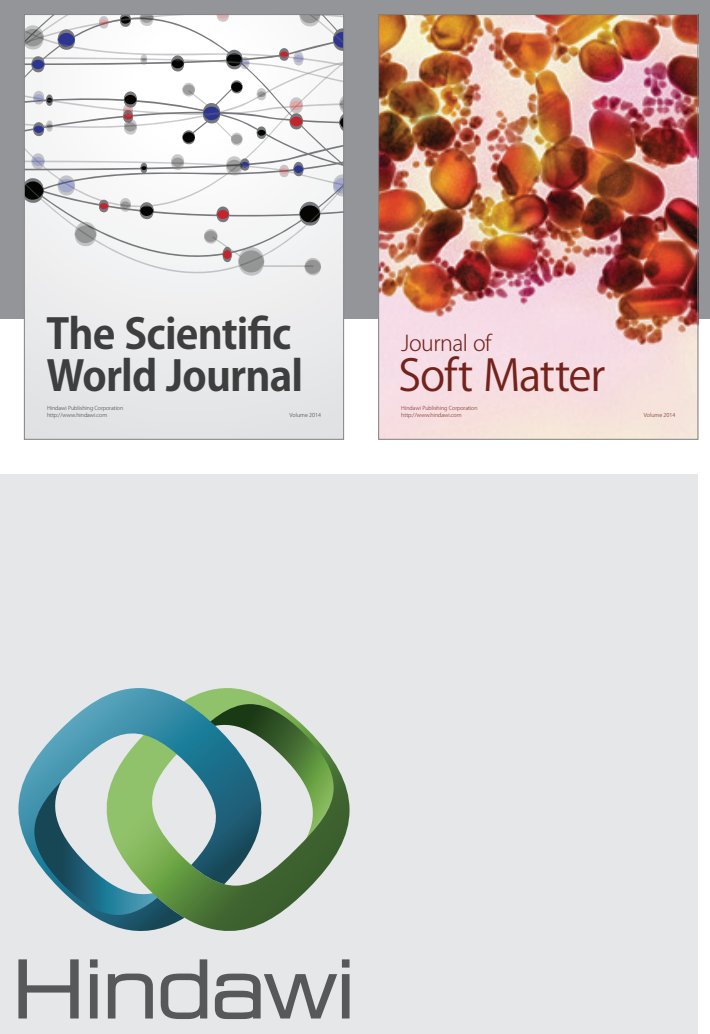

Submit your manuscripts at

http://www.hindawi.com
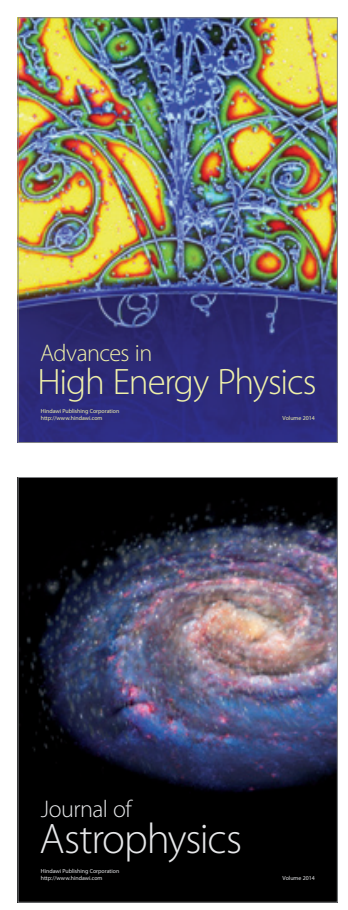
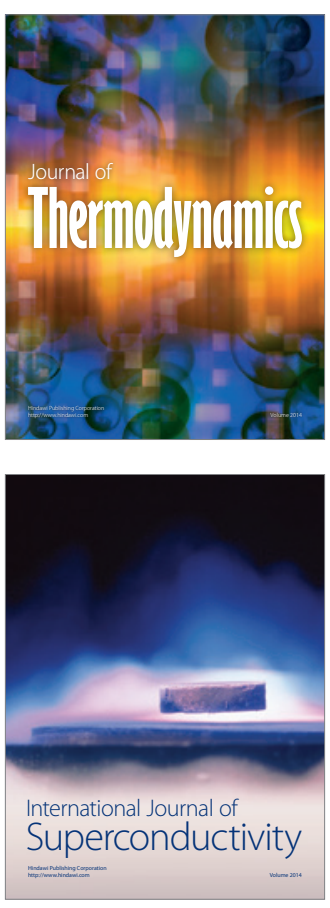
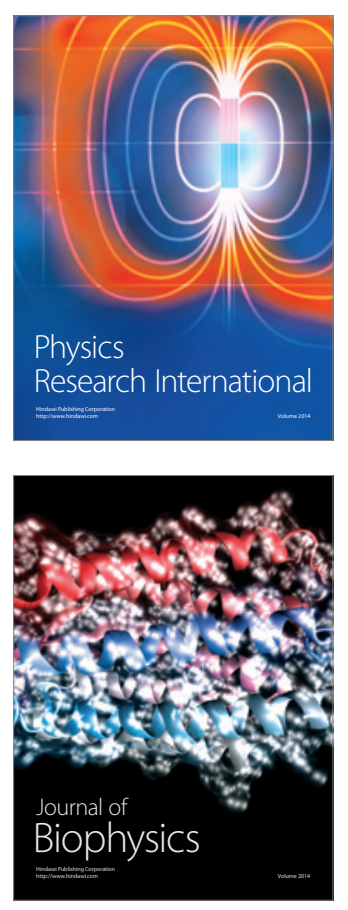
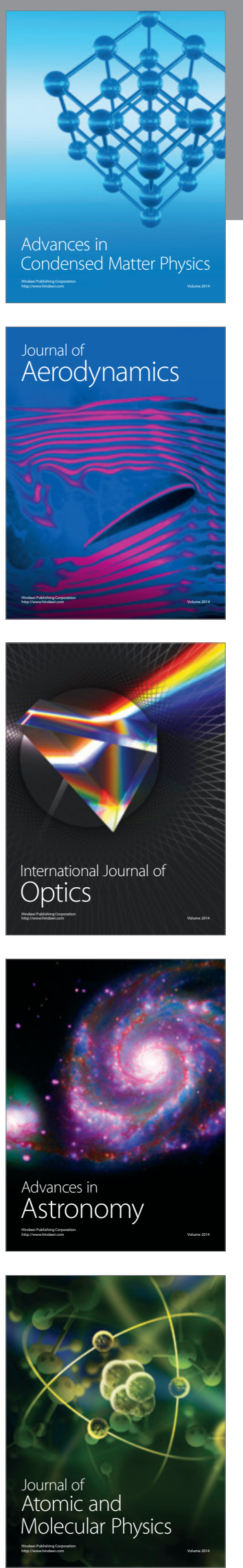